\title{
Use of Evidence-Centered Design to Develop Learning Maps-Based Assessments
}

\author{
Sue Bechard \\ Amy Clark* \\ Russell Swinburne Romine \\ Meagan Karvonen \\ Neal Kingston \\ University of Kansas \\ Karen Erickson \\ University of North Carolina \\ 1122 West Campus Road \\ Lawrence, KS 66045
}

The present publication was developed under grant 84.373 X100001 from the U.S. Department of Education, Office of Special Education Programs. The views expressed herein are solely those of the authors, and no official endorsement by the U.S. Department of Education should be inferred.

*Corresponding author

The Version of Record of this manuscript has been published and is available in International Journal of Testing, May 31, 2019 https://www.tandfonline.com/10.1080/15305058.2018.1543310 


\begin{abstract}
Evidence-based approaches to assessment design, development, and administration provide a strong foundation for an assessment's validity argument but can be time consuming, resource intensive, and complex to implement. This paper describes an evidence-based approach used for one assessment that addresses these challenges. Evidence-centered design principles were applied to create a task template to support test development for a new, instructionally embedded, large-scale alternate assessment system used for accountability purposes in 18 U.S. states. Example evidence from the validity argument is presented to evaluate the effectiveness of the template as an evidence-based method for test development. Lessons learned, including strengths and challenges, are shared to inform test-development efforts for other programs.

Keywords: evidence-centered design, test development, validity, next-generation assessments
\end{abstract}


Use of Evidence-Centered Design to Develop Learning Maps-Based Assessments

Evidence-centered design (ECD) describes a conceptual framework for designing, developing, and administering educational assessments that provide reliable, valid information about the targeted constructs (Mislevy, Steinberg, \& Almond, 1999). ECD requires test developers to describe the relationships between desired inferences about student knowledge, skills, and understandings and the tasks that can elicit evidence to support those inferences. The ECD approach is structured as a sequence of test-development layers that include domain analysis and modeling, conceptual assessment framework development, assessment implementation, as well as assessment delivery (Mislevy \& Riconscente, 2005). The use of ECD principles to design an assessment contributes to the validity argument because the process draws upon critical sources of evidence that are at the forefront of test design. These sources of evidence include test content, response processes, internal structure, relations to other variables, and consequences of testing (American Educational Research Association [AERA], American Psychological Association, \& National Council on Measurement in Education, 2014).

The benefits of using ECD as an approach to assessment development include supporting precise identification of task demands through the generation of design patterns, which can be used to create multiple items assessing the targeted knowledge, skills, and understandings. Design patterns are multifaceted and include a rationale; a description of focal knowledge, skills, and understandings; additional necessary, nonassessed knowledge, skills, and understandings; potential observations; potential work products; characteristic features of the assessment to evoke desired evidence; and variable features that can be adjusted for difficulty and focus (Riconscente, Mislevy, \& Corrigan, 2016). ECD also involves defining the intended uses of results at the outset, which informs each step of the test development process. 
While the theoretical benefits of ECD are well documented, the original processes pose numerous challenges for test developers. The method can be time consuming, resource intensive, and complex to implement (Ercikan, Seixas, Kaliski, \& Huff, 2016). As explicated by Hendrickson, Ewing, and Kaliski (2013) these challenges include: (a) integrating learning theory into assessment design; (b) identifying the appropriate levels of specificity for documenting claims and evidence; (c) developing and evaluating task models; and (d) strategically incorporating iterations into the design process. Describing observable evidence in the absence of a specific task can be difficult (Huff, Steinberg, \& Matts, 2010). It is therefore important to develop and evaluate test-design approaches that incorporate the principles of ECD and that attempt to overcome obstacles of implementation.

\section{Alternate Assessments Based on Alternate Achievement Standards}

Alternate assessments based on alternate achievement standards (AA-AAS) are largescale assessments used in the United States to ensure that students with the most significant cognitive disabilities have a way to participate meaningfully in statewide assessments used for accountability purposes (Karvonen, Wakeman, \& Kingston, 2017). These students are not defined by their disability labels but by IEP team determinations of their eligibility to take AAAAS. The learning characteristics of this population include slower rates of learning, limited communication systems (only $76 \%$ use speech to communicate), reduced short- and long-term memory capacities, difficulties making generalizations, and metacognitive challenges (Almond \& Bechard, 2005; Kleinert, Browder, \& Towles-Reeves, 2009; Nash, Clark, \& Karvonen, 2015). Because of these characteristics, students with significant cognitive disabilities require extensive, repeated, individualized instruction and support that is not of a temporary nature. Students with significant cognitive disabilities use substantially adapted materials and individualized methods 
of accessing information in alternative ways to acquire, maintain, demonstrate, and transfer skills across settings (Dynamic Learning Maps [DLM] Consortium, 2016).

Students with significant cognitive disabilities, who comprise approximately $1 \%$ of the $\mathrm{K}-12$ population and almost $10 \%$ of students with disabilities (Thurlow \& Wu, 2016), are expected to learn academic content aligned to grade-level content standards but with alternate achievement expectations. The academic achievement standards used in AA-AAS systems are connected to grade-level expectations for students in general education but at reduced depth, breadth, and complexity.

Historically, the formats for AA-AAS included teacher-created measures, such as bodyof-work, portfolios, teacher-administered performance tasks, rating scales or checklists, and combinations of these (Quenemoen, 2008). Teachers often selected the assessment content and determined which evidence constituted the student's skill mastery. The methods used in developing early AA-AAS typically did not match the technical rigor used for designing general education assessments (Bechard, 2005). In particular, the evidence needed to support the validity of inferences from AA-AAS scores was not well articulated. Following the adoption of ECD as a method for designing many general assessments, the principles and processes of ECD were extended for use in AA-AAS (DeBarger et al., 2011) to address the enormous variability in the population, the assumptions about measuring that population's achievement, and the variability of design-implementation procedures that made traditional assessment-design approaches inapplicable without some reformulation.

These efforts enhanced the development of ECD design patterns by integrating principles of Universal Design for Learning at multiple steps of the test-development process and describing ways to vary task features (e.g., adjusting difficulty) to support students in 
communicating what they understand and are able to do in relation to the focal knowledge, skills, and understandings.

This paper describes the design of a learning map (LM) model-based AA-AAS using principles of ECD. Specifically, it details an ECD approach designed to support efficient and effective test development, provides examples of evidence used to evaluate the effectiveness of the approach, and discusses strategies that address the challenges inherent in ECD usage. We also summarize strengths and challenges of the approach to inform application in other testing environments.

\section{Background of DLM Alternate Assessments}

AA-AAS should measure rigorous academic standards and assessment items should reflect relevant instructional activities and measure content students have had an opportunity to learn. DLM AA-AAS provide an innovative approach to measuring what students with significant cognitive disabilities know and can do in English language arts, mathematics, and science (Kingston, Karvonen, Bechard, \& Erickson, 2016). DLM offers two implementation models; the integrated model is described here, in which instructionally embedded, accessible assessments are administered throughout the year, with instruction and assessment occurring in tandem so that assessment results inform subsequent instructional decision-making. A spring assessment measures previously assessed content standards to update performance following the full year of instruction.

The architecture of the DLM assessment system is based on a framework of interconnected skills, called nodes, which are linked together by pathways indicating the order of skill acquisition. These complex networks are called learning map models. Maps are central to the DLM system's approach to the ECD domain-modeling process. The maps are based on 
multidisciplinary research syntheses that draw on instructional, cognitive, developmental, and academic subject-area literature. The maps provide a fine-grained representation of a content domain with multiple pathways toward development of targeted knowledge, skills, and understandings. The connections between nodes in the map are acyclic; precursor skills precede mastery of a learning target. Throughout the map model, there are multiple pathways leading to learning targets. Together, the skills and their prerequisite connections map out the progression of learning within a given domain.

While maps describe the pathways by which students develop expertise in an academic subject, the maps themselves are not defined by grade-specific expectations. The maps do include nodes that reflect content standards measured by the DLM system, called Essential Elements (EEs), which are the learning targets in English language arts and mathematics for grades 3-12. The EEs link the general education college- and career-ready content standards to grade-level expectations that are at an appropriate level of rigor and challenge for students with significant cognitive disabilities. The EEs specify end-of-year learning targets, while the DLM maps provide a rich representation of the multiple pathways that students can take to reach those targets.

Neighborhoods of nodes are identified for each EE at varying levels of cognitive complexity, called linkage levels. They serve as assessment targets and provide all students with significant cognitive disabilities an entry point for working toward rigorous grade-level academic expectations. For each EE, the five related linkage levels are identified in a specific region of the LM model, as shown in Figure 1. One linkage level includes the node(s) associated with the grade-appropriate target. Nodes at three precursor levels represent knowledge, skills, and understandings that precede mastery of the target, and nodes at the successor level measure the 
next steps toward the general education college and career ready standard. Nodes are measured by short assessments called testlets; each testlet includes an engagement activity and $3-9$ items measuring nodes at a single linkage level. At higher levels, a group of nodes may be assessed together to measure student ability to do more complex tasks that integrate content knowledge and procedural understanding. While the linkage level may measure more than one node, each individual item measures only a single node in the underlying map model. Most items are singleselect multiple choice items. However, when necessary to measure the node, multi-select multiple choice, drag and drop, click to place, select text, and matching line items are included.

[Figure 1]

Summative results, based on all assessments taken during the year, consist of the set of mastered skills for each subject. The DLM scoring model draws upon research in Bayesian inference networks and diagnostic classification modeling to determine linkage level mastery for each $\mathrm{EE}^{1}$. Separate latent class analyses are conducted to obtain the posterior probability of mastery, or the likelihood each student mastered the linkage level. A linkage level mastery threshold of .80 was determined from input from the governance board and Technical Advisory Committee and informed by data analyses. The value is far enough from .50 to ensure greater certainty in mastery classifications while also accounting for the historic variable performance of students with significant cognitive disabilities who take the assessment. An additional percent correct scoring rule ensures students who correctly respond to at least $80 \%$ of items measuring the linkage level are classified as masters. Mastery status is combined across linkage levels to determine the highest level mastered for each EE. Because students do not assess on all linkage

\footnotetext{
${ }^{1}$ For more information on the DLM approach to modeling, please see Chapter 5 of DLM Consortium, 2017.
} 
levels, mastery of a higher level assumes mastery of a lower level. For instance, mastery of the Target level assumes mastery of the three preceding linkage levels as well (see Figure 1).

\section{Incorporating ECD via Essential Element Concept Maps}

The DLM system uses a variant of ECD, derived from the DeBarger et al. (2011)

procedures, to create design patterns, development specifications, and task templates that include the essential features needed to build valid assessments for students with significant cognitive disabilities. These procedures include the application of Universal Design for Learning principles at multiple steps of the test-development process and allowances for the wide variety of supports students with significant cognitive disabilities need. For example, careful attention is given to the vocabulary students need in the context of the EE. DLM uses a comprehensive core vocabulary list, spanning grades $\mathrm{K}-12$, that reflects the research in core vocabulary in augmentative and alternative communication and words that students must express to demonstrate mastery of EEs (Dennis, Erickson, \& Hatch, 2013). These vocabulary words are provided in the task templates so that all students, regardless of their communication mode, can respond to items. In addition, considerations are made for students with vision, hearing, and/or mobility impairments, including development of assessments based on alternate map pathways or test-administration procedures. Several significant modifications to the DeBarger et al. (2011) model were made possible by the existence of the underlying map.

The DLM Consortium created and adopted task templates that were specifically designed for clarity and ease of use to support the project's nonprofessional item writers (typically classroom teachers) from participating consortium states. These templates, called Essential Element Concept Maps (EECMs), are graphic organizers that use the critical principles of ECD seen in design patterns, development specifications, and task templates to define assessment 
content for each testlet, as shown in Figure 2. The EECM provides information relevant to an assessment framework for one EE. The information is organized around the critical questions required by the ECD approach: (a) What is the student expected to know and do? What are we measuring? (b) What evidence is needed to determine if the student has the required knowledge, skills, and understandings? What are logical questions to ask to elicit observations of student performance? (c) What are potential tasks/products that can provide the evidence? How are varying levels of complexity addressed? and (d) How do we score student responses? (Bechard \& Sheinker, 2012). The content and descriptions included on each EECM are developed using Universal Design for Learning principles to prevent the unintended introduction of barriers to students who take DLM assessments.

[Figure 2]

The EECM is a concise organizational tool designed to make the complex representation of knowledge and skills in LM models accessible and useful for item writers. Each EECM includes an EE-specific local neighborhood mini-map derived from the larger LM model, as shown in Figure 3. The mini-maps provide a visual means of formally identifying the relationships between linkage levels, so item writers can consider the relationships during testlet design. Nodes may also be included in the mini-map as representations of important skills that may not be best assessed in a large-scale, standardized assessment. While these nodes might not be selected as an assessment target in a linkage level, their inclusion in the mini-map provides important information to item writers about the boundaries of content targeted for assessment. Because the mini-map provides a visual depiction and a description of the nodes surrounding the targeted skills, lengthy, textual descriptions of the nontargeted skills are not necessary. In applications of using a LM model for assessment development beyond DLM, a larger or smaller 
collection of nodes could be included in the mini-map to reflect coarser or more fine-grained measurement of the construct.

[Figure 3]

The distillation of the complexity of the LM structure into linkage levels for each EE addresses some challenges identified by Hendrickson et al. (2013) around integrating learning theory into assessment design and the need to provide appropriate levels of description of claims and evidence for test developers. Each EECM defines the content framework of the EE for the five linkage levels and identifies key concepts and vocabulary. At each linkage level, the EECM includes the LM node(s), a short description of the node, and a node observation that describes how evidence of student mastery of the node could be gathered. The node observations function similarly to the task models described by Mislevy, Almond, and Lukas (2003), describing the evidence to be collected. Each EECM also includes common misconceptions, questions to ask, and prerequisite and requisite skills. Finally, the EECM identifies potential accessibility challenges for students with sensory or physical limitations related to demonstration of the concepts being measured. Item writers are trained in the use of EECMs to support the development of testlets for each linkage level. In fact, item-development steps for item writers include familiarizing themselves with the EECM, using the EECM to plan a testlet, drafting the testlet, and finally checking the draft against the EECM before submitting the testlet for additional editorial, internal, and external reviews.

\section{Evidence Collected to Evaluate the Evidence-Centered Design Approach}

The process for evaluating the ECD-based approach to DLM test design draws upon much of the evidence collected to evaluate propositions in the assessment's validity argument. Table 1 summarizes the five sources of validity evidence used to evaluate the ECD-based 
approach, organized according to the critical sources of evidence indicated in the Standards for Educational and Psychological Testing (AERA, APA, \& NCME, 2014). The table also provides examples of how each source is evaluated, and specific data collected for evaluation. Additional details are provided in the following sections.

[Table 1]

\section{Test Content}

Evidence based on test content is collected to evaluate whether the assessment adequately measures the intended construct. This evidence is collected both before and after test administration and includes external review, measures of student opportunity to learn, alignment studies, and evaluation of map structure.

External review panels annually examine testlets prior to field-test administration. Panels look specifically at item-level criteria for content (alignment, depth of knowledge, quality and appropriateness, accuracy), accessibility (instructional relevance, clarity and appropriateness of images and graphics, minimizing barriers for students with specific needs), and bias and sensitivity (identifying items that require prior knowledge beyond the intended content or that are likely to elicit strong emotional response, ensuring fair representation of diversity, avoiding stereotypes, removing language that affects students' demonstration of their knowledge on the measurement target). Following review, panelists make a recommendation to accept content outright, revise content because of the violation of one or more criteria, or reject content when revision would not make the item acceptable. In 2017 , approximately $1 \%$ of content across grades, pools, and rounds of review was recommended for rejection (DLM Consortium, 2017), indicating support for the use of EECMs to produce testlets that are accessible, that measure the construct, and that are free from bias or sensitive content. 
Following test administration, an annual teacher survey is administered to evaluate students' opportunity to learn the assessed content. Because EECMs support the development of instructionally relevant testlets administered at instructionally relevant times, results from the teacher survey provide a broad source of evidence evaluating their effectiveness (see DLM Consortium, 2017).

An external alignment study was conducted after the first operational administration to investigate the relationships between content structures and assessment items, as illustrated in Figure 1. Because each of these relationships is considered in the ECD-based approach to DLM test development, alignment evidence can also be used to inform subsequent test-development efforts and potential revisions to EECM contents. As the ordering of linkage levels is fundamental to the design of DLM assessments, evidence was also collected during DLM pilot testing to evaluate whether the testlets reflected increasing difficulty. Students took fixed-form assessments composed of three testlets, each measuring a different linkage level for the EE. Overall, the percentage of correct responses decreased as the linkage level increased (Clark, Kingston, Templin, \& Pardos, 2014).

\section{Internal Consistency}

Because the assessment is calibrated and scored using a diagnostic model, results are reported as the profile of mastered skills (linkage levels) rather than a raw or scale score value. Internal consistency evidence is collected to evaluate how well the linkage levels collectively measure the construct. EECMs are designed to ensure that content measuring the same EE and linkage level is written to the same specifications. Therefore, all testlets measuring the same EE and linkage level are intended to be exchangeable. To evaluate the extent that item statistics support this assumption, the percentage of correct responses for each item is compared to the 
weighted average of all items measuring the EE and linkage level. Items are flagged for review by the test-development team when the value exceeds two standard deviations from the weighted mean. Items are similarly reviewed for evidence of differential item functioning (DIF), whereby items function differently for subgroups of the population (e.g., gender) after holding overall performance constant. Consistent with external review results for bias and sensitivity, less than 1\% of items were flagged for evidence of DIF in 2017 (DLM Consortium, 2017). Results from these analyses can also inform subsequent test-development efforts.

Parameters from the scoring model are also evaluated for consistency across all linkage levels. Because all items measuring the linkage level are assumed to be exchangeable, each linkage level is separately calibrated and the scoring model yields a single set of parameters for all items measuring the linkage level. Model parameters include the probability of providing a correct response to items measuring the linkage level for both masters and non-masters. When items perform as intended by the ECD-based approach, masters should have a high probability of providing a correct response and non-masters should have a low probability of providing a correct response. These assumptions were held for $98.3 \%$ of linkage levels for masters and $75.5 \%$ of linkage levels for non-masters. If these assumptions are not met, specific items, linkage levels, or the EECM may need to be reviewed. For example, if non-masters are likely to provide a correct response, test-development teams might consider whether the items measure something other than the nodes in the EECM or whether different nodes might provide a better measure of linkage level mastery for the EE.

\section{Response Process}

The aim of EECMs is to support item writers in the creation of testlets that elicit the intended cognitive process represented by the node. Evidence based on response process can be 
collected to evaluate how well the EECM fulfills this intention, through teacher-survey data, cognitive labs, and test-administration observations conducted by DLM staff and state and local education agency staff.

Following the 2017 administration, teacher-survey items gauged teacher perceptions of students' ability to respond to items as intended, free of barriers, and with necessary supports available. Most teachers agreed or strongly agreed that their students (a) responded to items to the best of their knowledge and ability (77.9\%); (b) were able to respond regardless of disability, behavior, or health concerns (65.8\%); and (c) had access to all supports necessary to participate (88.1\%; DLM Consortium, 2017). Because EECMs directly address accessibility considerations, including potential barriers for students who have vision, hearing, or mobility challenges, and because test administration accounts for accessibility features both within and outside of the test delivery system, high percentages of agreement are expected. Disagreement may reflect a lack of student engagement or possible local resource challenges for testlet administration.

Because DLM assessments use computer- and teacher-administered testlets in an online testing platform, response-process evidence must also evaluate the extent to which students respond as intended, particularly because of the heterogeneity of students with significant cognitive disabilities taking DLM assessments. Table 2 summarizes example behaviors observed during test-administration observations of computer-administered testlets. Evidence for teacheradministered testlets is collected to evaluate primary response mode and fidelity of response entry, as well as evidence of interrater agreement when scoring student work products. Although assessment items may be written to adequately measure the intended skills as defined in the EECM, if test administration does not accurately capture student responses, then inferences made from results may not be valid. 
[Table 2]

\section{Relation to Other Variables}

The ECD-based approach to test design described here is intended to adequately measure what students with significant cognitive disabilities know and can do. As such, linkage level mastery results from the DLM assessment should be related to other measures of students' knowledge, skills, and understandings and unrelated to variables such as demographic information (Mislevy et al., 2013). One source of external evidence examines the relationship between teachers' perceptions of student mastery of the skill measured by each EE and linkage level and the summative mastery decision from actual assessment results. To the extent the assessment adequately measures students' knowledge, skills, and understandings for each linkage level, the relationship should be strong. However, the correlation may be attenuated when teacher ratings do not accurately reflect what a student knows and can do. Since DLM assessments serve students with significant cognitive disabilities, many of whom have limited expressive communication systems, teacher ratings on some EEs and linkage levels may be more accurate than on others.

\section{Validity and Consequences of Testing}

DLM assessment results are intended to inform instructional practice. Evidence to evaluate their use is collected through teacher feedback via survey items, interviews, and focus groups, as well as through a score-report tutorial study that collects teacher feedback on the utility of score reports.

As reported, following the 2017 administration, teacher responses to survey items indicated general agreement that content reflected high expectations for their students (82\%), measured important academic skills (70\%), and was similar to classroom instructional activities 
(71\%). Although the majority of teachers expressed agreement, approximately $20 \%-30 \%$ did not. DLM assessments represent a departure from the breadth of academic skills assessed by many states' previous AA-AAS. Given the short history of general curriculum access for students with significant cognitive disabilities and the tendency for many teachers to prioritize instruction of functional academic skills (Karvonen, Wakeman, Browder, Rogers, \& Flowers, 2011), teachers' responses may reflect their awareness that DLM assessments contain challenging content rather than a limitation of test design. This assumption is being tested moving forward as evidence is gathered regarding teacher opportunity to learn via their completion of DLM-aligned professional development and associated perceptions of the testlets, the content they assess, and their appropriateness for students with significant cognitive disabilities.

\section{Discussion}

The DLM system incorporates LM models for domain modeling and EECMs as graphic organizers that incorporate a variant of ECD design patterns, task templates, and test specifications. Primary features of the EECM include Universal Design for Learning considerations, allowances for specific levels of cognitive complexity through linkage levels, and attention to the accessibility needs of students with significant cognitive disabilities. The intended outcome is an assessment designed to provide reliable and valid information about mastery of twenty-first-century academic skills for students who have historically been challenging to assess appropriately.

The benefits of the EECM as a tool to support an ECD-based approach include efficient test development by diverse item writers in a limited timeframe and the production of highquality items and testlets. The EECM helps ensure consistency in assessment content, internal 
consistency within the assessment system, and testlets that are accessible to students using diverse response processes. Operational use indicated low rates of rejection during external review and low flagging rates when analyzing for DIF.

Additionally, the use of EECMs provides an organized mechanism for iterative improvements to item-writing processes. Lessons learned in initial DLM development have informed subsequent item-writing events for DLM and other projects. When evidence indicated the EECM may not have supported effective item writing as intended, the content and/or the training in use of the EECM was revised. For example when testlet exchangeability is not observed it could be attributed to content of specific items or misconceptions on the part of the item writer; however, it may also be that changes to the EECM are required to ensure that testlets are written in a way that measures the construct as intended.

Multiple sources of evidence, including test content, response processes, internal structure, relations to other variables, and evidence for validity and consequences of testing, support the use of EECMs for developing instructionally embedded and relevant assessments of twenty-first-century skills in an AA-AAS for students with significant cognitive disabilities. When combined with procedural evidence ranging from research supporting the LM model structure to item-writer evaluations of their training and products, the entire body of evidence supports documentation for the technical adequacy of the DLM assessment system, consistent with professional standards (AERA, APA, \& NCME, 2014) and provides evidence in support of the ECD-based approach taken to develop the assessment. As the assessment system continues to mature, additional evidence can be collected. For example, statistical modeling will inform potential revisions to the LM models, and ongoing diagnostic classification modeling research 
may point to a need to change the assessment framework to strengthen claims about student mastery of EEs. 


\section{References}

American Educational Research Association, American Psychological Association, \& National Council on Measurement in Education. (2014). Standards for educational and psychological testing. Washington, DC: American Educational Research Association.

Almond, P., \& Bechard, S. (2005). In-depth look at students who take alternate assessments: What do we know now? Enhanced Assessment Grant project [Grant S368A03000]. Denver: Colorado Department of Education.

Bechard, S. (2005, April). Developing alternate assessments using expanded benchmarks from a nine state consensus framework in reading, writing, mathematics, and science. Paper presented at the annual meeting of the National Council on Measurement in Education, Montreal, Canada.

Bechard, S., \& Sheinker, A. (2012). Basic framework for item writers using evidence-centered design (ECD). Lawrence: University of Kansas.

Clark, A., Kingston, N., Templin, J., \& Pardos, Z. (2014). Summary of results from the fall 2013 pilot administration of the Dynamic Learning Maps ${ }^{\mathrm{TM}}$ Alternate Assessment System (Technical Report No. 14-01). University of Kansas Center for Educational Testing and Evaluation. Retrieved from http://dynamiclearningmaps.org/sites/default/files/documents/publication/pilot_summary of findings.pdf

DeBarger, A. H., Seeratan, K., Cameto, R., Haertel, G., Knokey, A-M., \& Morrison, K. (2011). Alternate assessment design-mathematics. Implementing evidence-centered design to develop assessments for students with significant cognitive disabilities: Guidelines for creating design patterns and development specifications and exemplar task templates for 
mathematics (Technical Report No. 9). Retrieved from

http://alternateassessmentdesign.sri.com/techreports/AAD_M_TechRpt9 032911final. pdf

Dennis, A., Erickson, K., \& Hatch, P. (2013). The Dynamic Learning Maps core vocabulary [Technical review]. DLM Professional Development Team, University of North Carolina at Chapel Hill. Retrieved from http://www.med.unc.edu/ahs/clds/files/vocabularyoverview

Dynamic Learning Maps Consortium. (2016). 2014-2015 technical manual-integrated model. University of Kansas, Center for Educational Testing and Evaluation. Retrieved from http://dynamiclearningmaps.org/sites/default/files/documents/publication/Technical_Man ual_IM 2014-15.pdf

Dynamic Learning Maps Consortium. (2017). 2016-2017 technical manual update_integrated model. University of Kansas, Accessible Teaching, Learning, and Assessment Systems (ATLAS). Retrieved from https://dynamiclearningmaps.org/sites/default/files/documents/publication/20162017 IM_Technical_Manual_Update.pdf

Ercikan, K., Seixas, P., Kaliski, P., \& Huff, K. (2016). Use of evidence-centered design in assessment of history learning. In H. Braun (Ed.), Meeting the challenges to measurement in an era of accountability (pp. 236-266). New York, NY: Routledge.

Hendrickson, A., Ewing, M., \& Kaliski, P. (2013). Evidence-centered design: Recommendations for implementation and practice. Journal of Applied Testing Technology, 14. Retrieved from https://atpu.memberclicks.net/assets/documents/evidencecentered $\% 20 \mathrm{design} \% 20 \mathrm{jatt} \% 20$ special $\% 20$ issue $\% 2013 . \mathrm{pdf}$ 
Huff, K., Steinberg, L., \& Matts, T. (2010). The promises and challenges of implementing evidence-centered design in large-scale assessment. Applied Measurement in Education, 23, 310-324. https://doi.org/10.1080/08957347.2010.510956

Karvonen, M., Wakeman, S. Y., Browder, D. M., Rogers, M. A. S., \& Flowers, C. (2011). Academic curriculum for students with significant cognitive disabilities: Special education teacher perspectives a decade after IDEA 1997. Retrieved from ERIC database: https://files.eric.ed.gov/fulltext/ED521407.pdf

Karvonen, M., Wakeman, S, \& Kingston, N.M. (2017). Alternate assessment. In M. L. Wehmeyer \& K. Shogren (Eds.) Handbook of research-based practices for educating students with intellectual disabilities (pp. 102-118). New York: Routledge.

Kingston, N. M., Karvonen, M., Bechard, S., \& Erickson, K. (2016). The philosophical underpinnings and key features of the Dynamic Learning Maps Alternate Assessment. Teachers College Record (Yearbook), 118(14). Retrieved from http://www.tcrecord.org

Kleinert, H. L., Browder, D. M., \& Towles-Reeves, E. A. (2009). Models of cognition for students with significant cognitive disabilities: Implications for assessment. Review of Educational Research, 79, 301-326. https://doi.org/10.3102/0034654308326160

Mislevy, R. J., Almond, R. G., \& Lukas, J. F. (2003). A brief introduction to evidence-centered design. ETS Research Report Series, 2003, i-29. doi:10.1002/j.23338504.2003.tb01908.x

Mislevy, R. J., Haertel, G., Cheng, B. H., Ructtinger, L., DeBarger, A., Murray, E., ... Vendlinski, T. (2013). A “conditional” sense of fairness in assessment. Educational Research and Evaluation, 19(2-3), 121-140. https://doi.org/10.1080/13803611.2013.767614 
Mislevy, R. J., \& Riconscente, M. (2005). Evidence-centered assessment design: Layers, structures, and terminology (Principled Assessment Designs for Inquiry Technical Report 9). Retrieved from https://padi.sri.com/downloads/TR9 ECD.pdf

Mislevy, R. J., Steinberg, L. S., \& Almond, R. G. (1999). Evidence-centered assessment design. Educational Testing Service. Retrieved from https://pdfs.semanticscholar.org/ebef/dc95626cf8467473b333eda5526ecd570dd9.pdf

Nash, B., Clark, A. K., \& Karvonen, M. (2015). First contact: A census report on the characteristics of students eligible to take alternate assessments (Technical Report No. 15-02). Lawrence: University of Kansas, Center for Educational Testing and Evaluation.

Quenemoen, R. (2008). A brief history of alternate assessments based on alternate achievement standards (Synthesis Report 68). University of Minnesota, National Center on Educational Outcomes. Retrieved from https://nceo.umn.edu/docs/OnlinePubs/Synthesis68/Synthesis68.pdf

Riconscente, M. M., Mislevy, R. J., \& Corrigan, S. (2016). Evidence-centered design. In S. Lane, S., M. R. Raymond, \& T. M. Haladyna (Eds.), Handbook of test development (pp. 4063). New York, NY: Routledge.

Rupp, A. A., Templin, J., \& Henson, R. A. (2010). Diagnostic measurement: Theory, methods, and applications. New York, NY: The Guilford Press.

Thurlow, M., \& Wu, Y.-C. (2016). 2013-2014 APR snapshot \#12: AA-AAS participation and performance. University of Minnesota, National Center on Educational Outcomes. Retrieved from https://nceo.info/Resources/publications/APRsnapshot/brief12/index.html 


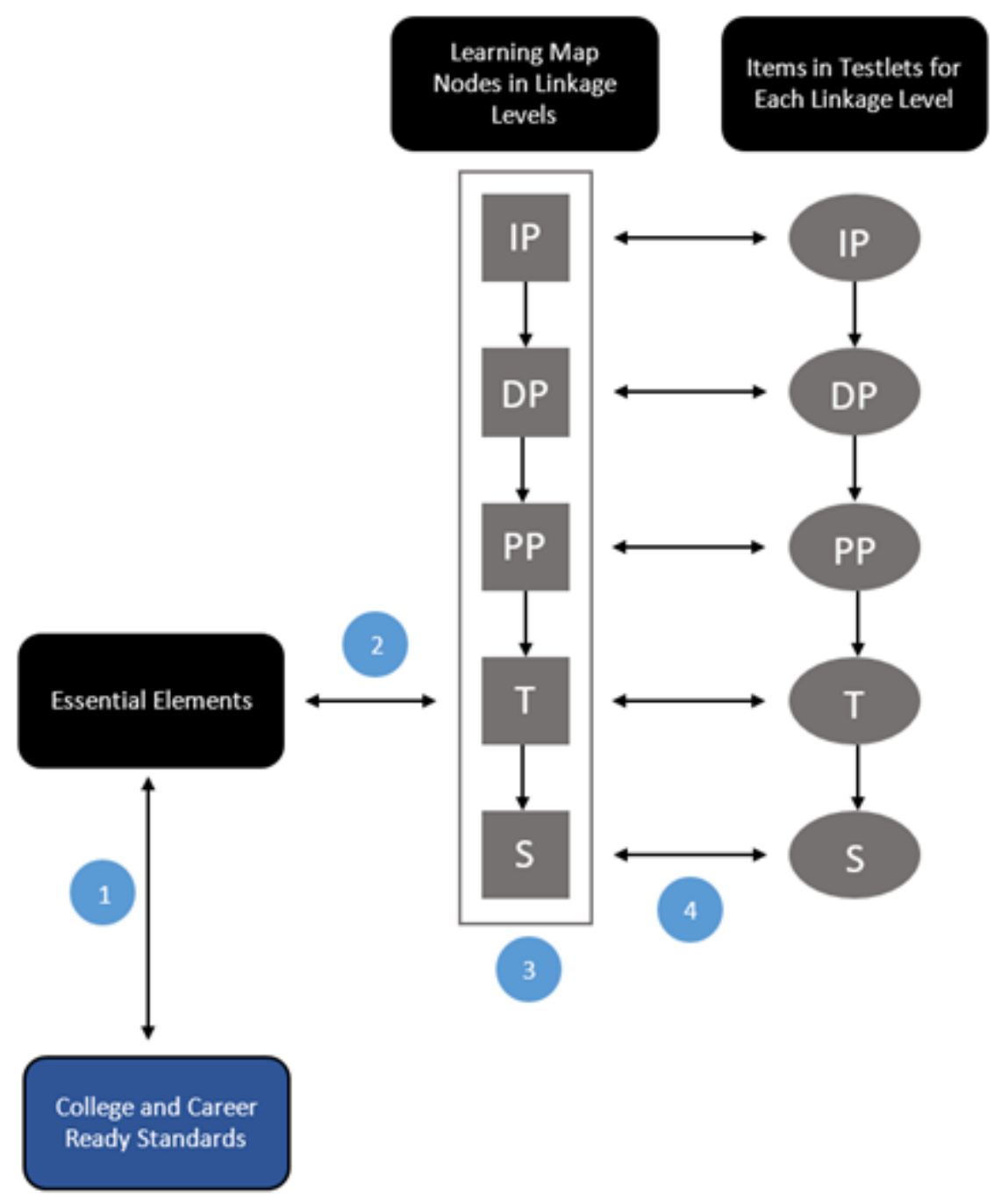

Figure 1. Relationships in the DLM Alternate Assessment System, including (1) College and Career Ready Standards and Essential Elements, (2) an Essential Element and its target node(s), (3) vertical articulation of linkage levels, and (4) assessment items and nodes they measure. Linkage levels are Initial Precursor (IP), Distal Precursor (DP), Proximal Precursor (PP), Target (T), and Successor (S). 


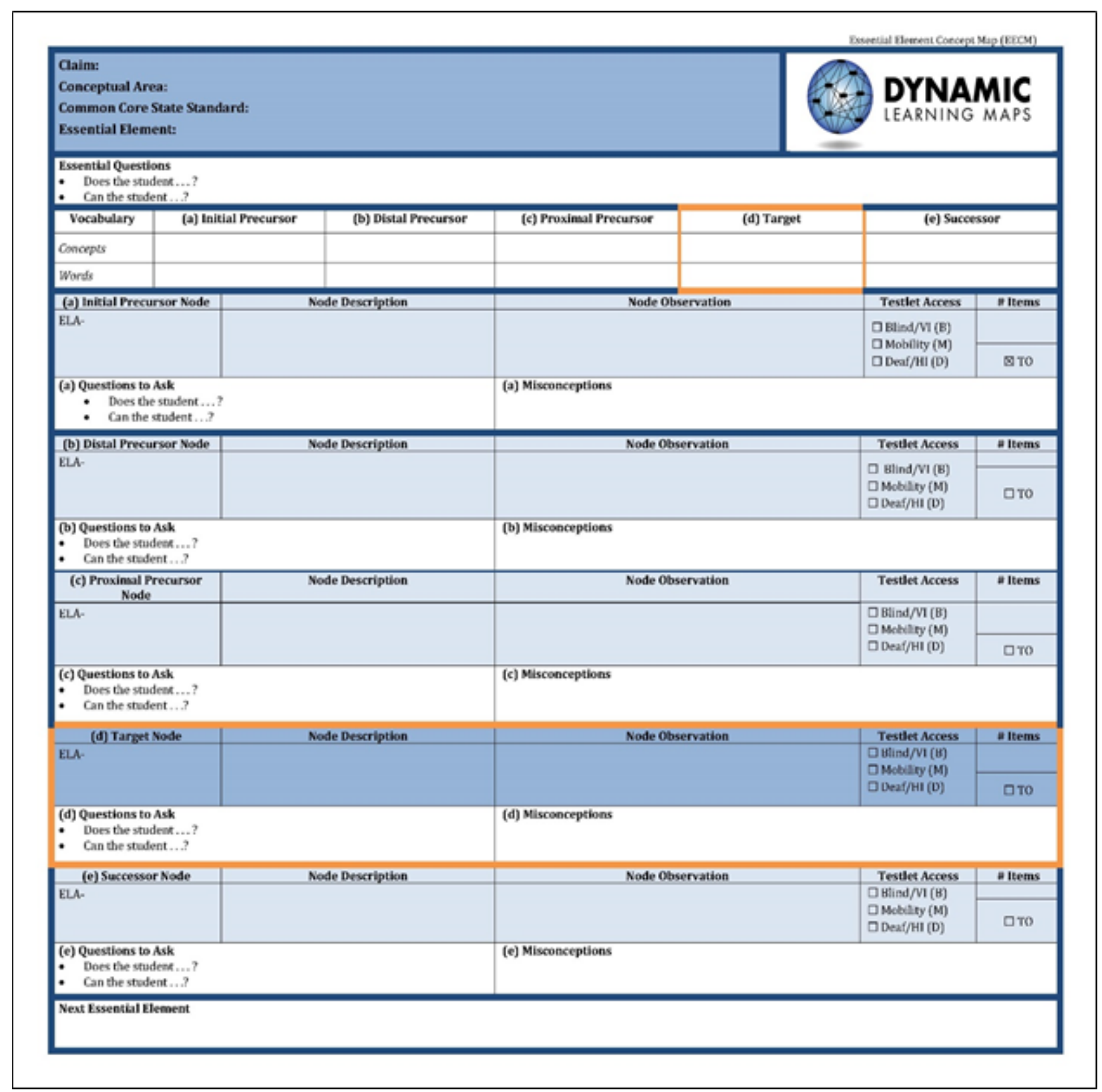

Figure 2. Example Essential Element Concept Map graphic organizer. 


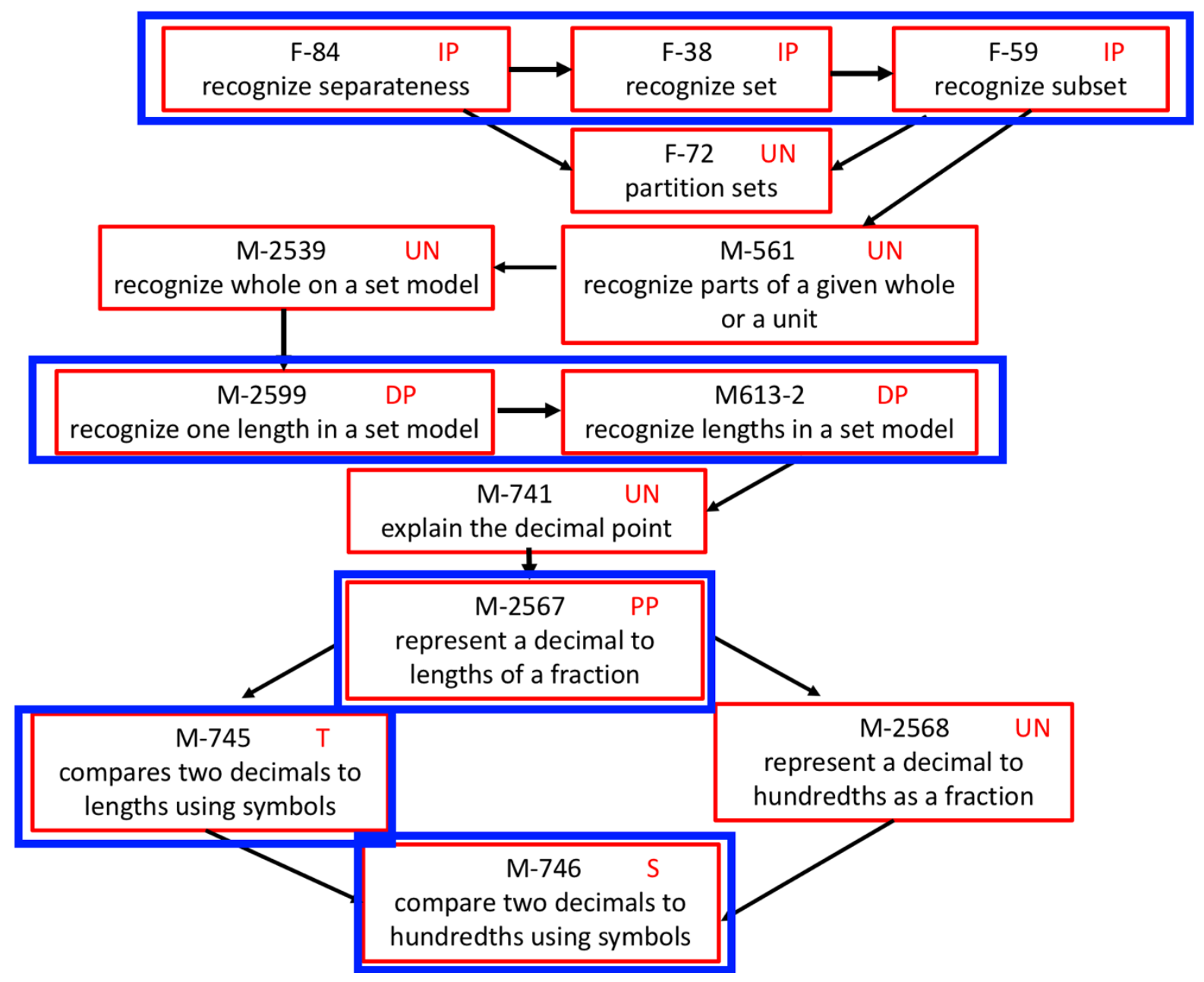

Figure 3. Example mathematics mini-map for Essential Element 7.NS.3—“Compare quantities represented as decimals in real world problems to tenths." Thick boxes indicate node(s) measured in each linkage level. Linkage levels are labeled in the upper-right corner. IP $=$ Initial Precursor; DP $=$ Distal Precursor; $\mathrm{PP}=$ Proximal Precursor; $\mathrm{T}=$ Target; $\mathrm{S}=$ Successor; $\mathrm{UN}=$ untested node. 
Table 1

Evaluation and Data Collection for Sources of Validity Evidence

\begin{tabular}{|c|c|c|}
\hline Evidence & Evaluation & Data collection \\
\hline \multirow{5}{*}{ Test content } & Alignment & $\begin{array}{l}\text { Internal review } \\
\text { External panels }\end{array}$ \\
\hline & Instructional relevance & Teacher survey \\
\hline & Opportunity to learn & Teacher survey \\
\hline & Order of linkage levels & Student response data \\
\hline & External review & $\begin{array}{l}\text { Accessibility, bias and sensitivity, } \\
\text { and content panels }\end{array}$ \\
\hline Internal consistency & $\begin{array}{l}\text { Exchangeability of } \\
\text { testlets }\end{array}$ & $\begin{array}{l}\text { Student response data } \\
\text { Model parameters }\end{array}$ \\
\hline Response process & Respond as intended & $\begin{array}{l}\text { Test-administration observations } \\
\text { Cognitive labs } \\
\text { Teacher survey }\end{array}$ \\
\hline \multirow[t]{3}{*}{ Relation to other } & Related to measures of & Teacher ratings correlated with \\
\hline & knowledge, skill, ability & mastery \\
\hline & Unrelated to descriptives & Disability status \\
\hline
\end{tabular}


Demographic data

$\begin{array}{lll}\text { Validity and } & \text { Focus groups } \\ \text { consequences of } & \text { Use results as intended } & \text { Interviews } \\ \text { testing } & & \text { Teacher feedback on tutorial }\end{array}$


Table 2

Test Administrator Actions During Computer-Administered Testlets $(N=102)$

\begin{tabular}{|c|c|c|c|}
\hline Evidence & Action & $n$ & $\%$ \\
\hline \multirow{5}{*}{ Supporting } & Read one or more screens aloud to the student & 51 & 50.0 \\
\hline & Clarified directions or expectations for the & 51 & 50.0 \\
\hline & \multicolumn{3}{|l|}{ student } \\
\hline & Navigated one or more screens for the student & 30 & 29.4 \\
\hline & Repeated question(s) before student responded & 40 & 39.2 \\
\hline \multirow{9}{*}{ Neutral } & Asked the student to clarify or confirm one or & 14 & 13.7 \\
\hline & more responses & \multirow{3}{*}{5} & \multirow{3}{*}{4.9} \\
\hline & Repeated question(s) after student responded & & \\
\hline & (i.e., gave a second trial at the same item) & & \\
\hline & Allowed student to take a break during the testlet & 17 & 16.7 \\
\hline & Used verbal prompts to direct the student's & 44 & 43.1 \\
\hline & attention or engagement (e.g., "look at this") & & \\
\hline & Used pointing or gestures to direct student & 29 & 28.4 \\
\hline & Used materials or manipulatives during the & 12 & 11.8 \\
\hline
\end{tabular}


to the student

Nonsupporting

Physically guided the student's hand to an

answer choice

Note. Respondents could select multiple responses. 